TITLE:

\title{
Mean field theory for the intermolecular and intramolecular conformational transitions of a single flexible polyelectrolyte chain
}

$\operatorname{AUTHOR}(\mathrm{S})$ :

Shew, Chwen-Yang; Yoshikawa, Kenichi

\section{CITATION:}

Shew, Chwen-Yang ...[et al]. Mean field theory for the intermolecular and intramolecular conformational transitions of a single flexible polyelectrolyte chain. Journal of Chemical Physics 2007, 126(14): 144913.

ISSUE DATE:

2007-04-14

URL:

http://hdl.handle.net/2433/45688

\section{RIGHT:}

Copyright (2007) American Institute of Physics. This article may be downloaded for personal use only. Any other use requires prior permission of the author and the American Institute of Physics. 


\title{
Mean field theory for the intermolecular and intramolecular conformational transitions of a single flexible polyelectrolyte chain
}

\author{
Chwen-Yang Shew ${ }^{\text {a) }}$ \\ Department of Chemistry, College of Staten Island and Graduate Center, City University of New York, \\ Staten Island, New York 10314 \\ Kenichi Yoshikawa \\ Department of Physics, Graduate School of Science, Kyoto University, Kyoto 606-8502, Japan
}

(Received 30 October 2006; accepted 13 February 2007; published online 13 April 2007)

\begin{abstract}
The diMarzio theory has been extended to elucidate the intermolecular and intramolecular phase segregations of a single flexible chain polyelectrolyte in dilute salt-free solutions. At the long chain limit, this theory yields the formalism obtained from the more sophisticated Edward Hamiltonian for polyelectrolyte problems. The calculated phase diagram exhibits the features of a first-order phase transition, with continuous and discontinuous transitions separated by a critical point. Under the discontinuous transition, the polyelectrolyte chain exhibits coexistent expanded and collapsed conformational states, same as intermolecular phase segregation. For a limiting long chain, the mean chain size at critical point is roughly $90 \%$ of the size of an ideal chain. Such a result implies that partial contraction within a chain molecule is required to collapse a flexible polyelectrolyte chain. Moreover, the theory predicts that for a longer chain, intramolecular segregated conformations differ significantly from intermolecular segregated conformations, but the difference becomes small for shorter chains. Besides, the charge needed to induce intramolecular segregation is smaller than that of intermolecular segregation for a given chain length. These findings are consistent with previous literature results. (C) 2007 American Institute of Physics. [DOI: 10.1063/1.2714552]
\end{abstract}

\section{INTRODUCTION}

The coil-globule conformational transition of a single flexible polymer molecule has been one of the most fundamental problems in polymer physics. ${ }^{1,2}$ For single flexible chain molecules without electronic charge, their coil-globule transition is known to be continuous as solvent quality is varied. ${ }^{2,3}$ Whereas the conformational behavior of an isolated flexible polyelectrolyte in solution seems to remain unclear. Theoretical predictions and simulations have suggested that the conformational transition of an isolated flexible charged polymer could be discontinuous, with coexistent collapsed and expanded conformational states as in the first-order phase transition. ${ }^{4-7}$ Such a study is crucial to advance our understanding of more complex ionic polymers, such as DNA of which conformational transition governs the switching of transcriptional activities on-and-off in vivo. ${ }^{8,9} \mathrm{Al}$ though the discrete nature of the coil-globule transition of a giant DNA chain has been mainly described in terms of semiflexibility of the chain molecule, ${ }^{10}$ the role of electrostatic interaction on the conformational behavior on both semiflexible and flexible chains needs to be investigated further. In this work, our focus will be confined to the electrostatic effect on a flexible chain.

The coil-globule conformational transition of a single charged polymer with the bimodal free energy profile on monomer density may undertake intermolecular and intramolecular phase segregations. ${ }^{11-13}$ The intermolecular

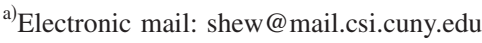

phase segregation is referred to the discrete conformational transition between disperse and condensed states, i.e., coexisting (bistable) coil and globule states, where individual polymer molecules exhibit either state. This type of transition is as opposed to the continuous transition where monomer density changes in a gradual manner as in the textbook explanation on the transition through theta state. In the experiment, intermolecular segregation is characterized via measuring the coexistent globular and coiled conformational states. ${ }^{14}$ A histogram is obtained to quantify the probability of each chain size in different conformational states. The coexistent states are best observed when the probability of both states is near the same. The conditional free energy as a function of mean chain size can be estimated from the histogram.

In the intramolecular phase segregation, a polymer chain consists of both coiled segments and globule parts. The structure may range from a chain containing one globule and one coiled segment to a pearl necklace chain with several charged pearls connected by coiled segments. ${ }^{11-13}$ Figure 1 is the schematic diagram of the possible pathways for intermolecular and intramolecular segregations. Experimentally, these chain structures have been observed in both semiflexible long chain DNA molecules ${ }^{15-18}$ and synthetic flexible polyelectrolytes. ${ }^{12,19-21}$ In the mean time, the intramolecular segregated conformations have been extensively investigated in recent theoretical studies and simulations. ${ }^{722-25}$ Among these theoretical works, Dobrynin et al. ${ }^{22}$ explained the origin of pear-necklace-like structures based on the Rayleigh instability $^{26}$ by which a globular polyelectrolyte chain in 


$$
\alpha^{5}-\alpha^{3}=\alpha^{-3}-\tau N^{1 / 2}+\frac{u f^{2}}{b} N^{3 / 2} \alpha^{2},
$$

where $\alpha$ is the swelling ratio $\left(=R / R_{0}\right)$, where $R$ and $R_{0}$ are the mean chain sizes of a polyelectrolyte and an ideal chain, respectively; $\tau$ is a parameter related to solvent quality and temperature; $u$ is the Bjerrum length; $f$ is the monomer charge fraction; $b$ is the monomer length; and $N$ is the chain length. After solving the above equation numerically, they attained the range of $f$ where the globular and coiled conformational states coexist in the plot of $f$ vs $\alpha$ for a given chain length $N$ and solvent quality $\tau$. They argued that the intramolecular segregated conformations, such as pearl necklace chain structure, is located within this region since such a structure lies in between the coiled and globular conformational states. Note that this picture is consistent with the simulation work by Limbach et al. regarding the highly fluctuating nature of the pearl necklace chain. ${ }^{24}$ Also, they suggested a systematic scheme to estimate the intramolecular phase segregation boundary in the plot of $f$ vs $\alpha$ for a given $N$. The critical monomer charge fraction to induce intramolecular phase segregation for each solvent condition could be obtained by using Maxwell equal-area construction. At this critical monomer charge fraction, the splitting of the collapsed chain occurs because the electrostatic repulsive force balances with the line tension of the polymer chain. ${ }^{31,32}$

All of the above mentioned works under poor solvents were targeted only on the part of the phase diagram to illustrate the discrete conformational transition of single chain polyelectrolytes. To the best of our knowledge, the complete phase diagram and the critical behavior of the conformational transition of a single polyelectrolyte molecule have not been addressed systematically from the standpoint of theory and experiment. In this work, we are motivated to construct the conformational phase diagram of a single flexible polyelectrolyte molecule under different solvent qualities through the mean field theory suggested by diMarzio. ${ }^{33,34}$

The diMarzio theory is a revision of the theory developed by Flory, which incorporates the excluded volume interaction and the polymer-solvent interactions into the single chain free energy at the mean field level. Previously, Shew and Yethiraj applied this theory for single flexible chain polyelectrolytes and compared with lattice simulations. They found that the theory reproduces the correct power laws obtained from other theoretical approaches, such as scaling theory. ${ }^{6}$ Moreover, the theory predicts the first-order phase transition for the conformational transition of a flexible chain polyelectrolyte in poor solvents, consistent with the simulations. Here, we will first show that this simple theory yields the formalism in Eq. (1) for long enough chains. With this equation, the chain length dependent phase diagram and the critical behavior are examined. Given that all of the actual polymer molecules are finite systems, the chain length dependent studies may render an opportunity to extrapolate the behavior of an infinity long chain and to compare with other known phase transition systems. In this work, both intermolecular and intramolecular phase segregations are investigated through the calculated phase diagram. 


\section{THEORY}

\section{A. Free energy}

Here we consider the simplest polyelectrolyte model consisting of a flexible charged chain immersed in an extremely dilute solution by using the diMarzio model. diMarzio revisited the Flory-type theory for the conformation of a neutral isolated polymer chain in different solvent conditions. The polymer molecule is treated as a Gaussian chain with monomer length equal to $b$, and the excluded volume and solvent effects are considered in a similar way as the Flory-Huggins solution theory. ${ }^{33,34}$ The weighing function of a chain conformation is written as a function of mean chain size $R$ (e.g., mean end-to-end distance) given by

$$
\begin{aligned}
W(R)= & {\left[R^{2} \exp \left(-\frac{3 R^{2}}{2 N b^{2}}\right)\right]\left[\Pi_{j=0}^{n-1}\left(1-\frac{j b^{3}}{R^{3}}\right)\right] } \\
& \times\left\{\exp \left[-N \chi\left(1-\frac{N b^{3}}{R^{3}}\right)\right]\right\},
\end{aligned}
$$

where $N$ is the chain length; $\chi$ denotes solvent qualities in which $\chi=1 / 2$ for $\theta$ solvent, $\chi<1 / 2$ for good solvents, and $\chi>1 / 2$ for poor solvents. The argument with $\chi$ parameter accounts for the total monomer-solvent pairs, with 1 $-N b^{3} / R^{3}$ volume fraction of solvent particles occupied within the entire volume of a chain molecule. For charged polymers, the electrostatic interaction energy of an ionic chain is reformulated as a function of mean chain size and is approximated to be $u f^{2} N^{2} / R$, which can be found in the previous works by Shew and Yethiraj ${ }^{6}$ and by deGennes et al. ${ }^{35}$ In this work, the counterions are not incorporated explicitly because under the extremely dilute condition, entropically, it is more favorable for counterions to totally dissociate from polyions. Furthermore, this model is chosen to compare with the model based on the Edwards Hamiltonian. ${ }^{31}$

After some algebra, the weighing function of mean chain size $W(\alpha)$ as a function of swelling ratio $\alpha(=R / \sqrt{N} b)$ becomes

$$
\begin{aligned}
\ln (\alpha W(\alpha))= & \ln \alpha^{3} N^{3 / 2}-\frac{3}{2} \alpha^{2}-N \chi\left(1-N^{-1 / 2} \alpha^{-3}\right) \\
& +\alpha^{3} N^{3 / 2} \ln \left(\alpha^{3} N^{3 / 2}\right) \\
& -\left(\alpha^{3} N^{3 / 2}-N\right) \ln \left(\alpha^{3} N^{3 / 2}-N\right)-N \\
& -N \ln \left(\alpha^{3} N^{3 / 2}\right)-3 \Gamma \frac{N^{3 / 2}}{\alpha}
\end{aligned}
$$

where $\Gamma=u f^{2} / 3$ in which $u$ is the Bjerrum length and $f$ is the monomer charge fraction. According to the suggestion of diMarzio, the optimal mean chain dimension should be evaluated by maximizing the distribution function $\ln [\alpha W(\alpha)]$ other than $\ln [W(\alpha)]$ to maintain the correct coefficients as Flory's result for the mean squared end-to-end distance of an ideal chain. ${ }^{33}$ This argument is equivalent to taking free energy to be $F / k_{B} T=-\ln (\alpha W(\alpha))$. This free energy expression is a conditional free energy with the variable $\alpha$, which can be obtained from the experimental histogram for chain size distribution. ${ }^{14}$ By minimizing the free energy (i.e., $d F / d \alpha$ $=0$ ), we arrive at

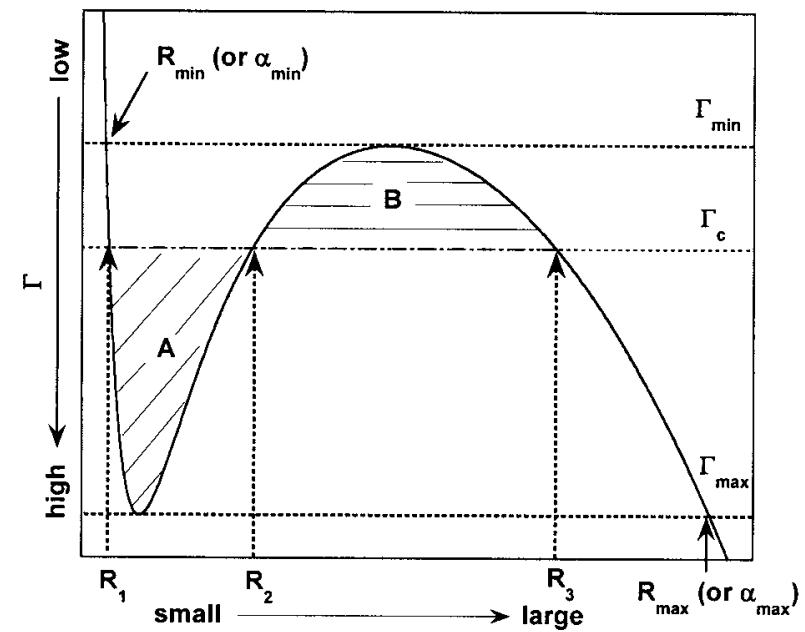

FIG. 2. Schematic plot for illustration of the Maxwell equal-area construction.

$$
\begin{aligned}
\alpha^{5}-\alpha^{3}= & -\alpha^{6} N^{3 / 2}\left[\ln \left(1-N^{-1 / 2} \alpha^{-3}\right)+N^{-1 / 2} \alpha^{-3}\right] \\
& -N \chi+\Gamma N^{3 / 2} \alpha^{2} .
\end{aligned}
$$

The solutions of the above equation lead to optimal mean chain sizes. It has been shown that this equation generates the correct power laws for neutral chains in different solvent qualities and for polyelectrolytes in good and $\theta$ solvents, compared to those obtained from more sophisticated methods and scaling theory. ${ }^{6,33}$

\section{B. Construction of phase boundary}

In this work, two methods are employed to construct phase boundary, including free energy method introduced in Sec. II A and Maxwell equal-area construction. In the schematic plot of Fig. 2, the curve ( $\Gamma$ vs $\alpha$ ) represents the solutions of Eq. (4) for a poor enough solvent with coexistent conformational states. Note that $\Gamma$ increases from top to bottom and $\alpha$ (or $R$ ) increases from left to right in the figure. Between $\Gamma_{\min }$ and $\Gamma_{\max }$, there are three solutions of $\alpha$ for the given $\chi$. With Eq. (4), the intermolecular phase segregation boundary is constructed in such a way that the two coexistent conformations in different conformational states exhibit the same free energy. Experimentally, these two coexistent conformations would display equal probability in the histogram for the chain size distribution. ${ }^{14}$

Beside intermolecular segregation, intramolecular segregation can also be evaluated in Fig. 2. For $\Gamma<\Gamma_{\min }$, the chain molecule is in the collapsed state, whereas for $\Gamma>\Gamma_{\max }$, the chain molecule is in its coiled state. The intramolecular segregation should emerge in the range between $\Gamma_{\min }$ and $\Gamma_{\max }$. The chain size of intramolecular segregated conformation should be between $\alpha_{\min }$ and $\alpha_{\max }$, corresponding to collapsed globule and extended coil, respectively. As pointed out by Dua and Vilgis, Maxwell equal-area construction provides an estimation of the critical charge to induce the spontaneous Rayleigh splitting. ${ }^{31}$ The rationale is given as follows. In Fig. 2 the critical charge $f_{c}$ (or $\Gamma_{c}=u f_{c}^{2} / 3$ ) is the dividing line to keep the area in regions A and B the same. The equal-area criterion is equivalent to the following equation: 


$$
\int_{R_{1}}^{R_{2}}\left(\Gamma-\Gamma_{c}\right) d \alpha=\int_{R_{2}}^{R_{3}}\left(\Gamma_{c}-\Gamma\right) d R
$$

where all the parameters are defined in Fig. 2. With some algebra, $\Gamma_{c}$ reads

$$
\Gamma_{c}=\frac{1}{\left(R_{3}-R_{1}\right)} \int_{R_{1}}^{R_{3}} \Gamma d R .
$$

This equation can be further rearranged by using the following steps:

$$
\begin{aligned}
\Gamma_{c} & =\frac{1}{\left(R_{3}-R_{1}\right)} \int_{R_{1}}^{R_{3}}\left(\frac{\Gamma}{R^{2}}\right) R^{2} d R \\
& =\left[\frac{\left(V_{3}-V_{1}\right)}{4 \pi\left(R_{3}-R_{1}\right)}\right]\left[\frac{1}{\left(V_{3}-V_{1}\right)} \int_{V_{1}}^{V_{3}}\left(\frac{\Gamma}{R^{2}}\right) d V\right] \\
& =\frac{R_{3}^{2}}{3}\left[1+\left(\frac{R_{1}}{R_{3}}\right)+\left(\frac{R_{1}}{R_{3}}\right)^{2}\right]\left\langle\frac{\Gamma}{R^{2}}\right\rangle,
\end{aligned}
$$

where $V=4 \pi R^{3} / 3$, by assuming spherelike morphology for a chain molecule. ${ }^{36}$ Since the volume ratio of $V_{3}$ to $V_{1}$ is larger than the order of $10^{4}$ in experiments (i.e., $\left.R_{3} \gg R_{1}\right), \Gamma_{c}$ $\approx\left(R_{3}^{2} / 3\right)\left\langle\Gamma / R^{2}\right\rangle$. Meanwhile, the mean electrostatic repulsive force of the polymer is related to the critical charge fraction $f_{c}$ in the expression given by

$$
k_{B} T \frac{N^{2} u f_{c}^{2}}{R_{n l}^{2}}=\left\langle k_{B} T \frac{N^{2} u f^{2}}{R^{2}}\right\rangle,
$$

where $R_{n l}^{2}=R_{3}^{2} / 3$. Now $\Gamma_{c}$ and $R_{n l}$ are located between the globular and elongated coiled states. In poor solvents, the collapsed globule starts to split at the point where the mean Coulomb repulsive force balances with line tension $\left(k_{B} T \tau / b\right) .{ }^{31,32}$ This critical charge can be viewed as the inception of intramolecular segregation due to Rayleigh instability. Although it is not our main focus, the two balanced

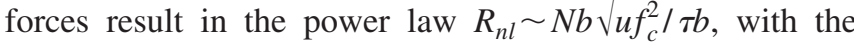
characteristics of intramolecular segregated conformations, such as pearl necklace structure, as in Ref. 31. With these two methods, the discrimination between intermolecular and intramolecular segregations is performed based on the chain length dependent phase boundaries.

\section{Critical constants}

For a given chain length $N$, the phase diagram is governed by three variables: $\alpha, \chi$, and $\Gamma$. To complete the phase diagram, the critical point is calculated from $d \chi / d \alpha=0$ and $d^{2} \chi / d \alpha^{2}=0$ given by

$$
\begin{aligned}
N^{1 / 2} \frac{d \chi}{d \alpha}= & -6 \alpha^{5} N^{3 / 2}\left[\ln \left(1-N^{-1 / 2} \alpha^{-3}\right)+N^{-1 / 2} \alpha^{-3}\right] \\
& -3 \alpha^{2} N\left[\frac{1}{\left(1-N^{-1 / 2} \alpha^{-3}\right)}-1\right] \\
& +2 \Gamma N^{3 / 2} \alpha+3 \alpha^{2}-5 \alpha^{4}
\end{aligned}
$$

$$
\begin{aligned}
N^{1 / 2} \frac{d^{2} \chi}{d \alpha^{2}}= & -30 \alpha^{4} N^{3 / 2}\left[\ln \left(1-N^{-1 / 2} \alpha^{-3}\right)+N^{-1 / 2} \alpha^{-3}\right] \\
& -24 \alpha N\left[\frac{1}{\left(1-N^{-1 / 2} \alpha^{-3}\right)}-1\right] \\
& +9 \frac{N^{1 / 2} \alpha^{-2}}{\left(1-N^{-1 / 2} \alpha^{-3}\right)^{2}}+2 \Gamma N^{3 / 2}+6 \alpha-20 \alpha^{3}
\end{aligned}
$$

After eliminating $\Gamma$, we obtain

$$
\begin{aligned}
5 \alpha^{5} & -\alpha^{3}+8 \alpha^{6} N^{3 / 2}\left[\ln \left(1-N^{-1 / 2} \alpha^{-3}\right)+N^{-1 / 2} \alpha^{-3}\right] \\
& +7 \alpha^{3} N\left(\frac{1}{1-N^{-1 / 2} \alpha^{-3}}-1\right)-\frac{3 N^{1 / 2}}{\left(1-N^{-1 / 2} \alpha^{-3}\right)^{2}}=0 .
\end{aligned}
$$

After solving the swelling ratio at the critical point $\left(\alpha^{*}\right)$ by using Eq. (11), the critical constant of solvent quality $\left(\chi^{*}\right)$ is obtained from Eq. (9) or (10), and $\Gamma^{*}$ is calculated through Eq. (4). These equations are nontrivial for our analysis of the long chain limit. Note that the critical point can also be calculated from $d \Gamma / d \alpha=0$ and $d^{2} \Gamma / d \alpha^{2}=0$, and the critical constants are exactly the same for both methods.

\section{Long chain limit}

When $N$ becomes large, the above equations can be simplified furthermore. For large $N, \ln \left(1-N^{-1 / 2} \alpha^{-3}\right)$ is approximated to be $-N^{-1 / 2} \alpha^{-3}-N^{-1} \alpha^{-6} / 2+O(N)$, where $O(N)$ denotes the higher order terms. With this approximation, Eq. (4) is simplified to the following form:

$$
\alpha^{5}-\alpha^{3}=\frac{\alpha^{-3}}{3}-\left(\chi-\frac{1}{2}\right) N^{1 / 2}+\left(\Gamma N^{3 / 2}\right) \alpha^{2} .
$$

This equation is basically the same as Eq. (1) by Dua and Vilgis, ${ }^{31}$ except the prefactor in the $\alpha^{-3}$ term (from the higher order approximation of excluded volume interaction). Note that as a matter of fact, Eq. (1) has also been derived by Ha and Thirumalai, and the prefactor of the $\alpha^{-3}$ term was treated as an adjustable parameter in their work. ${ }^{5}$

Moreover, by using Taylor expansion, Eq. (11) becomes

$$
\alpha^{8}-\frac{\alpha^{6}}{5}-\frac{1}{3}=0
$$

The solution of this equation can be computed numerically with a high precision to obtain the critical constant $\alpha^{*}$, and $\alpha^{*} \approx 0.902901$ when $N \rightarrow \infty$. Such a result has an important implication on the chain conformation at near the critical point but has not been investigated in the previous work by Shew and Yethiraj. ${ }^{6}$ With similar algebra, the limiting $\chi^{*}$ becomes

$$
\left(\chi^{*}-\frac{1}{2}\right) N^{1 / 2}=\frac{1}{2}\left(3 \alpha^{* 5}-\alpha^{* 3}+\frac{5}{3} \alpha^{*-3}\right)+N^{-1 / 2} \alpha^{*-6}+O(N),
$$



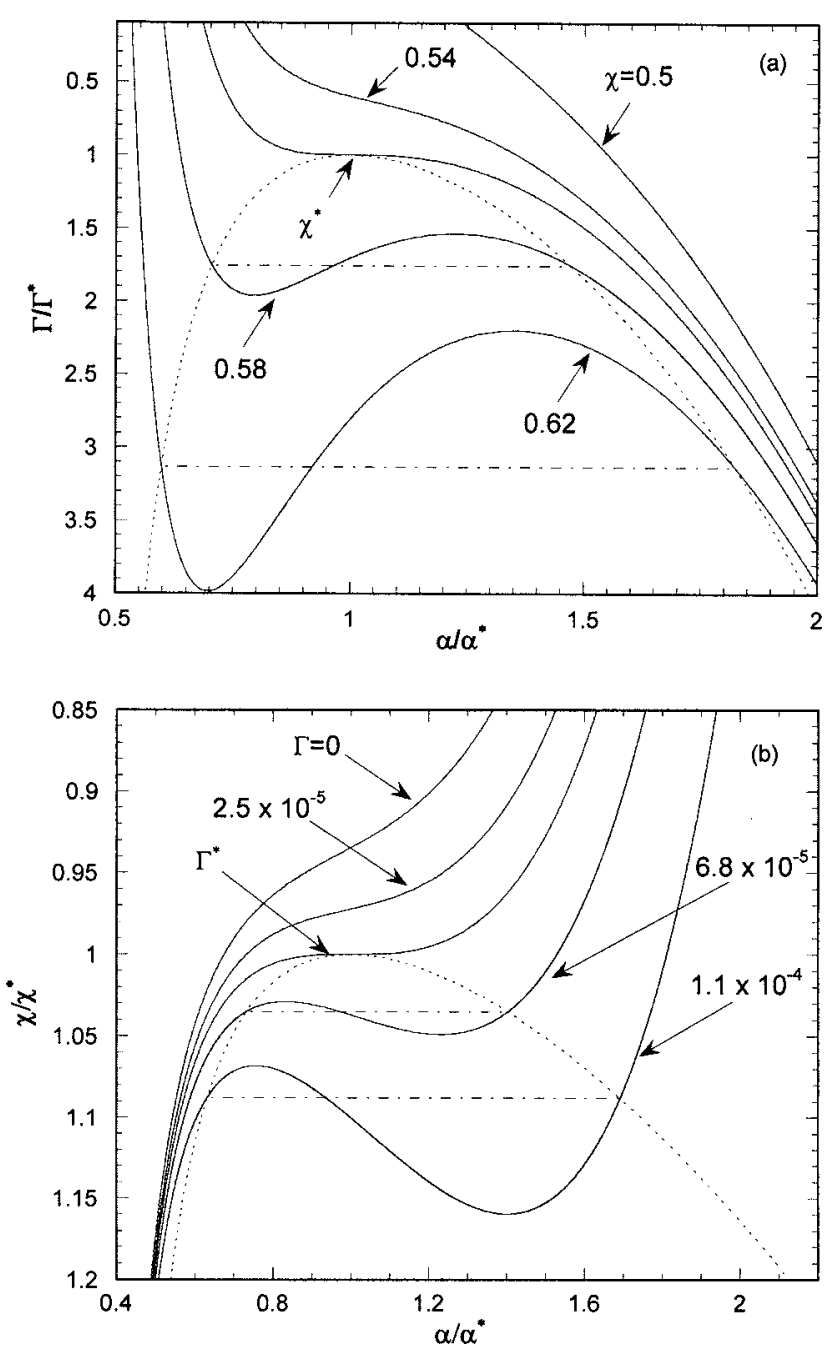

FIG. 3. Plot of the variation of $\Gamma / \Gamma^{*}$ with $\alpha / \alpha^{*}$ in (a) for different $\chi$, as marked, and the variation of $\chi / \chi^{*}$ with $\alpha / \alpha^{*}$ in (b) for different $\Gamma$, as marked, when $N=1000$, as well as the corresponding phase boundaries, denoted by dotted lines.

$$
\Gamma^{*} N^{3 / 2}=\left(\frac{5}{2} \alpha^{* 3}-\frac{3}{2} \alpha^{*}+\frac{4}{3} \alpha^{*-5}\right)+\frac{3}{4} N^{-1 / 2} \alpha^{*-8}+O(N) .
$$

The above two equations yield the same power laws as in the previous work by Shew and Yethiraj, ${ }^{6}$ i.e., $\left(\chi^{*}-1 / 2\right)$ $\sim N^{-1 / 2}$ and $\Gamma^{*} \sim N^{-3 / 2}$.

\section{RESULTS AND DISCUSSION}

\section{A. Intermolecular phase segregation}

The phase diagram is first constructed by solving Eq. (4) along with the equal free energy method, and the thus obtained phase boundary is related to the coexistent coilglobule states of intermolecular segregation. Figure 3 plots the variation of $\Gamma / \Gamma^{*}$ with $\alpha / \alpha^{*}$ in (a) for different $\chi$, as marked, and the variation of $\chi / \chi^{*}$ with $\alpha / \alpha^{*}$ in (b) for different $\Gamma$, as marked, when $N=1000$, as well as the corresponding phase boundaries, denoted by dotted lines. Both figures show continuous conformational transitions above the critical point. In Fig. 3(a), $\alpha$ increases monotonically (from left to right) as $\Gamma$ is increased (from top to bottom) for a given $\chi$ because of chain expansion induced by the Cou-
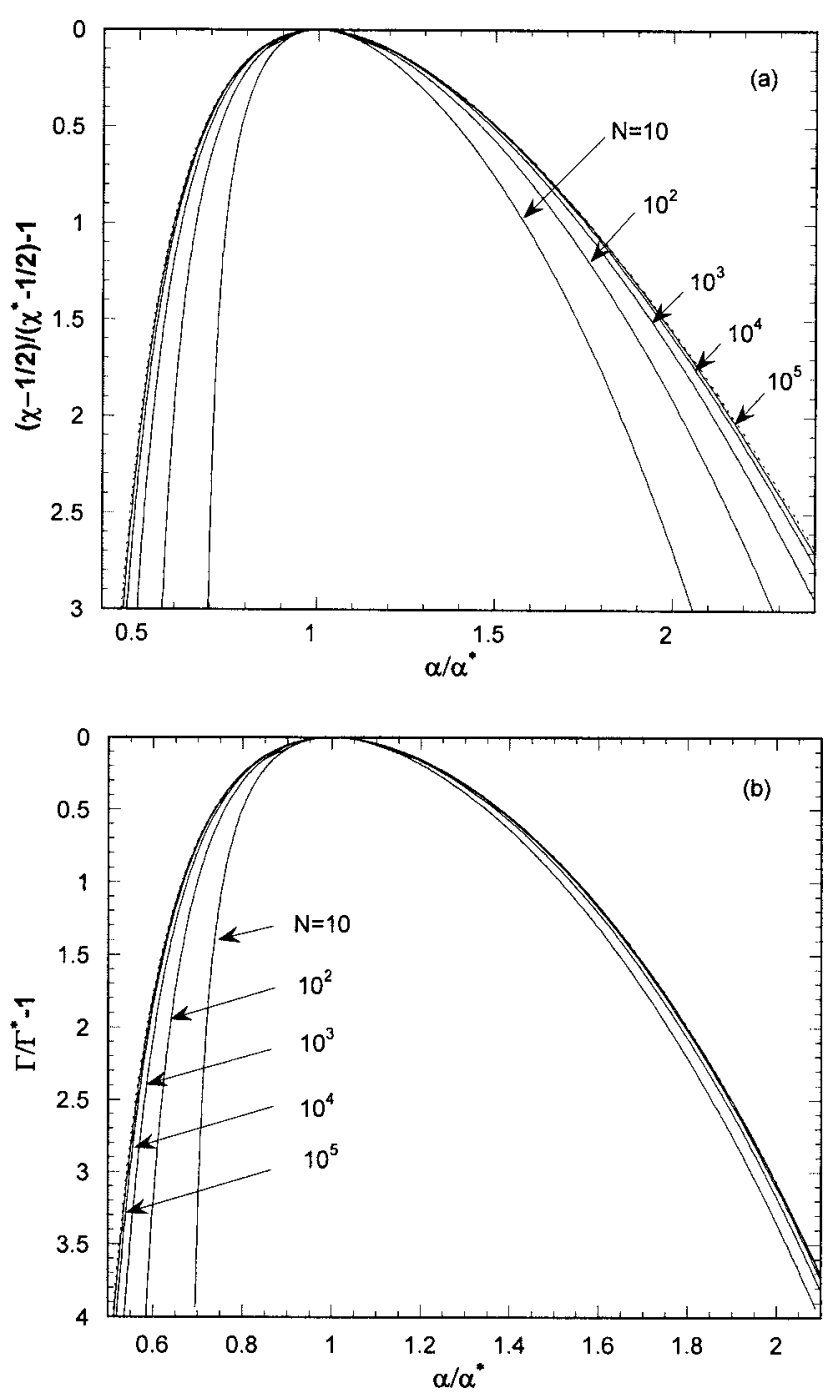

FIG. 4. Plot of the phase boundary in terms of solvent quality $[(\chi$ $\left.-1 / 2) /\left(\chi^{*}-1 / 2\right)-1\right]$ vs swelling ratio $\left(\alpha / \alpha^{*}\right)$ in (a) and electrostatic interaction strength $\left(\Gamma / \Gamma^{*}\right)$ vs swelling ratio $\left(\alpha / \alpha^{*}\right)$ in (b) for different chain lengths $N$, as marked, including $N=\infty$, denoted by dotted lines.

lomb repulsion between monomers. In Fig. 3(b), $\alpha$ decreases monotonically (from right to left) as $\chi$ is increased (from top to bottom) for a given $\Gamma$ due to chain contraction when the solvent quality is decreased. Below the critical point, the region of coexisting conformational states emerges in the phase diagram, with three solutions of $\alpha$ for a given $\chi$ in Fig. 3 (a) and for a given $\Gamma$ in Fig. 3(b). The phase boundary is obtained when the minimum and maximum $\alpha$, among the three solutions, have the same free energy (connected by dash-dotted lines in Fig. 3).

The chain length dependence on the intermolecular phase segregation boundary is further calculated. In Fig. 4, the phase boundary is plotted in terms of solvent quality $\chi$ versus swelling ratio $\alpha$ in (a) and electrostatic interaction strength $\Gamma$ versus swelling ratio $\alpha$ in (b) for different chain lengths $N$, as marked, including $N=\infty$, denoted by dotted lines. The phase boundary is asymmetric in both plots. In contrast to globular state, coiled state deforms more as $\chi$ or $\Gamma$ is increased (from top to bottom). For shorter chains, such as $N=10$, the phase boundary is narrower than that of a longer chain. As the chain length is increased, the width only in- 


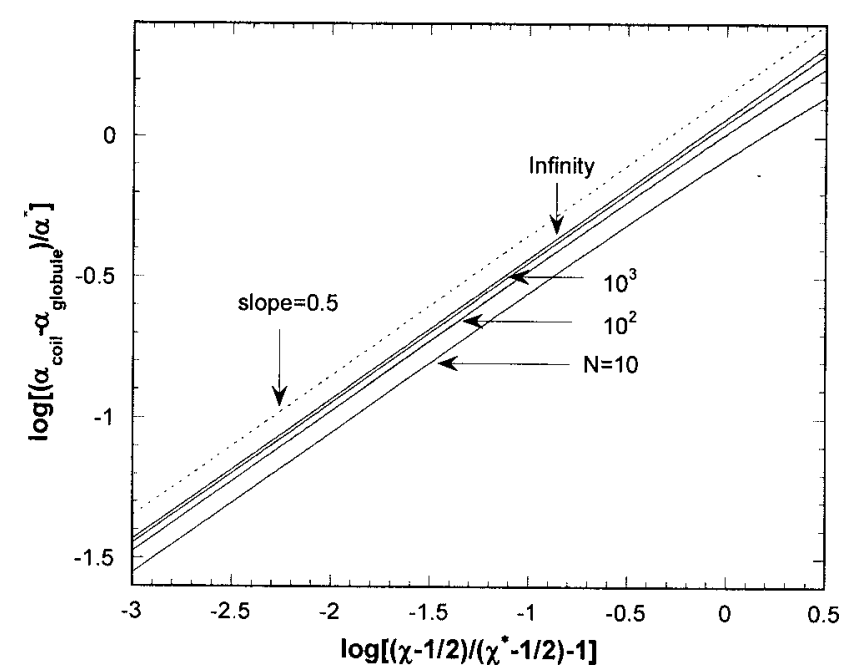

FIG. 5. Plot of $\log \left[\left(\alpha_{\text {coil }}-\alpha_{\text {globule }}\right) / \alpha^{*}\right]$ vs $\log \left[(\chi-1 / 2) /\left(\chi^{*}-1 / 2\right)-1\right]$ for different chain lengths $N$; the dotted line denotes the slope obtained from linear regression.

creases slightly, and the phase boundary becomes insensitive to chain length in the range of our plot when $N$ is larger than $10^{4}$. It is noticeable that at near the critical point, the phase boundary is quite independent of chain length.

With the above phase diagrams, the critical behavior is investigated by computing the power laws: $\left(\alpha_{\text {coil }}-\alpha_{\text {globule }}\right)$ $\sim\left[(\chi-1 / 2) /\left(\chi^{*}-1 / 2\right)-1\right]^{\beta_{1}} \quad$ and $\quad\left(\alpha_{\text {coil }}-\alpha_{\text {globule }}\right)$ $\sim\left[\left(\Gamma-\Gamma^{*}\right) / \Gamma^{*}\right]^{\beta_{2}}$. Figure 5 displays the plot of $\log \left[\left(\alpha_{\text {coil }}\right.\right.$ $\left.\left.-\alpha_{\text {globule }}\right) / \alpha^{*}\right]$ vs $\log \left[(\chi-1 / 2) /\left(\chi^{*}-1 / 2\right)-1\right]$ for different chain lengths $N$; dotted lines denote the slopes obtained from linear regression. Our finding shows that $\beta_{1}=\beta_{2}=\beta=0.5$ (the figure for $\beta_{2}$ not shown) and are insensitive to chain lengths. These results imply that the diMarzio theory falls into the mean field universality class. ${ }^{37}$ It is not surprising to obtain such an exponent since we expect that the conformational fluctuation is not adequately considered in the mean field model. Nevertheless, this exponent provides a reference point for the future work in this area. At present, it is obvious that the exponent $\beta$ obtained from experiments should be smaller than or equal to 0.5 because the scaling exponent $\beta$ of the mean field universality class is at the upper bound among all universality classes.

In the following, the chain length dependent critical constants are revisited. Figure 6 displays the variation of the critical constants $\chi^{*}$ in (a), $\Gamma^{*}$ in (b), and $\alpha^{*}$ in (c) with chain length $N$. For long enough chains, $\chi^{*}$ in Fig. 6(a) and $\Gamma^{*}$ in Fig. 6(b) exhibit the power laws predicted by Eqs. (14) and (15), respectively. These scaling relations indicate that at the long chain limit, the discrete conformational transition occurs when solvent quality is slightly below $\theta$-solvent condition and/or monomers are weakly charged. In Fig. 6(c), the critical mean chain size decreases monotonically as $N$ is increased, and eventually, it becomes leveled off at the long chain limit. The limiting swelling ratio $\alpha^{*}$ for $N=\infty$ is obtained from Eq. (13), which is approximately equal to 0.902 901. This limiting swelling ratio is less than 1 , meaning that the chain conformation tends to collapse partially at the critical point such that the mean chain size is smaller than that of an ideal chain. The partial contraction within a chain
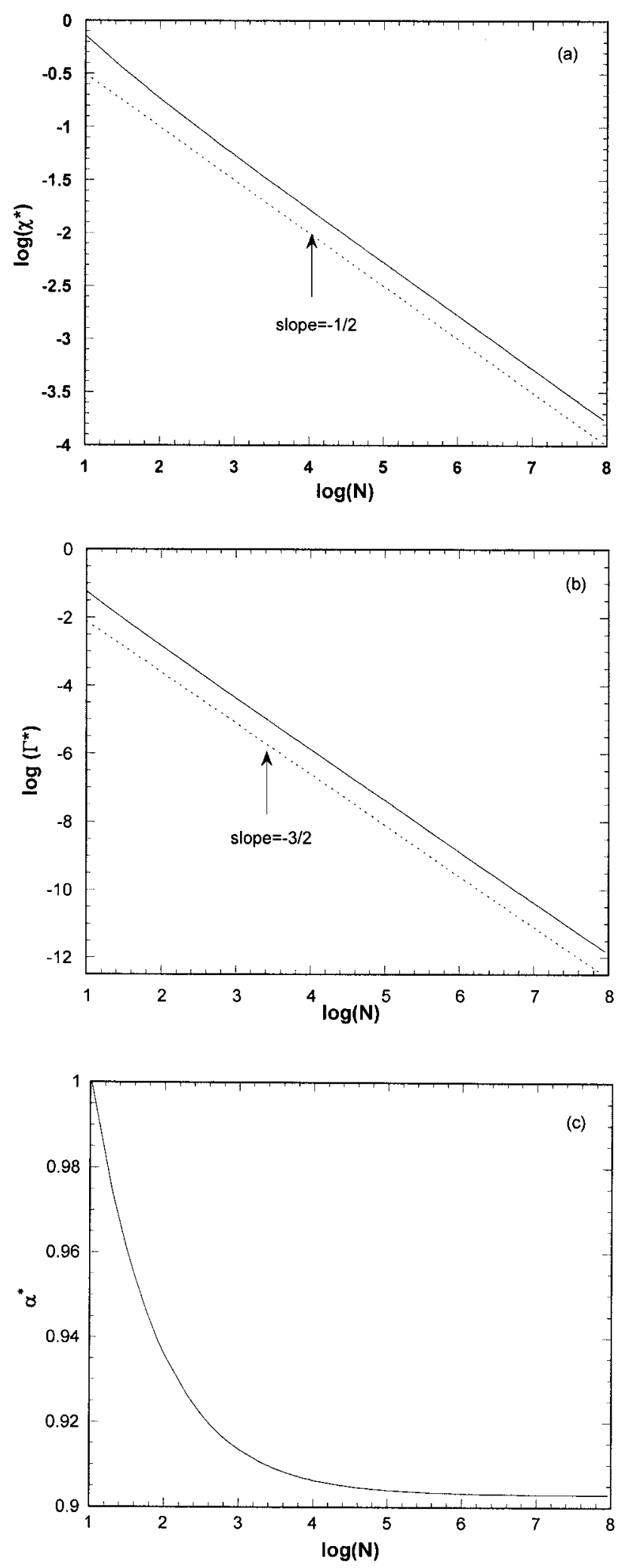

FIG. 6. Variation of the critical constants $\chi^{*}\left(\log -\log\right.$ plot) in (a), $\Gamma^{*}(\log -\log$ plot) in (b), and $\alpha^{*}$ (semilog) in (c) with chain length $N$.

molecule, predicted by the diMarzio model, may link with the folding of a flexible polyelectrolyte chain. ${ }^{38,39}$

\section{B. Intramolecular phase segregation}

In addition to intermolecular phase segregation, this simple mean field model may also provide some insights into 


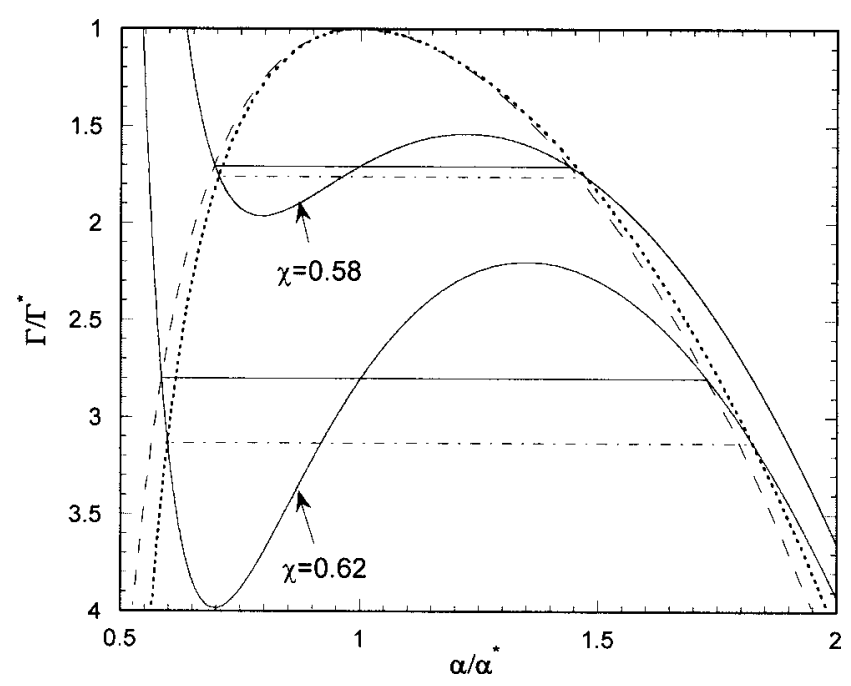

FIG. 7. Comparison of the phase boundary obtained from the Maxwell construction method, denoted by broken line, with that obtained from the equal free energy method, denoted by dotted line, for $N=1000$. Two examples, $\chi=0.58$ and 0.62 , are shown to illustrate the critical charge fraction for spontaneous Rayleigh splitting through Maxwell construction, denoted by solid lines, and the two bistable conformations of equal free energy connected by dotted broken lines.

intramolecular phase segregation. As discussed in Sec. II B, intramolecular segregated conformations lie in between the coiled and globular conformational states. ${ }^{31}$

The critical charge fraction required for the spontaneous Rayleigh splitting of a charged globule (into intramolecular segregated conformations) is estimated by using Maxwell equal-area construction. ${ }^{31}$ Figure 7 plots the phase boundary obtained from the Maxwell construction method, denoted by broken line, and that obtained from the equal free energy method, denoted by dotted line. In the figure, two examples, $\chi=0.58$ and 0.62 , are shown to illustrate how the critical charge fraction for the spontaneous Rayleigh splitting and the corresponding phase boundary are computed through Maxwell construction. These calculations are further compared with the results from the equal free energy method. In Fig. 7, the horizontal solid lines divide the close loops with two equal areas (by using Maxwell construction), whereas the horizontal dotted broken lines connect the two bistable conformation states (of equal free energy). Generally, for a given $\chi$, the charge fraction of solid line occurs at a smaller $\Gamma$ than that of dotted broken line (e.g., for $\chi=0.62, \Gamma / \Gamma^{*}$ $=2.80$, denoted by solid line, and 3.13 , denoted by dotted broken line), suggesting that intramolecular segregation occurs at a lower charge fraction than intermolecular segregation.

The general picture for the phase diagram can be understood as follows. For a small monomer charge fraction, the polyelectrolyte chain forms a globule in a poor solvent condition. As the monomer charge fraction is increased to the critical charge (estimated from Maxwell construction), the globule reaches the Rayleigh instability, and the formation of intramolecular segregated conformations is expected. When the monomer charge is increased further, the polyelectrolyte molecule attains the bistable coil-globule conformational states (with equal free energy for the two conformations).

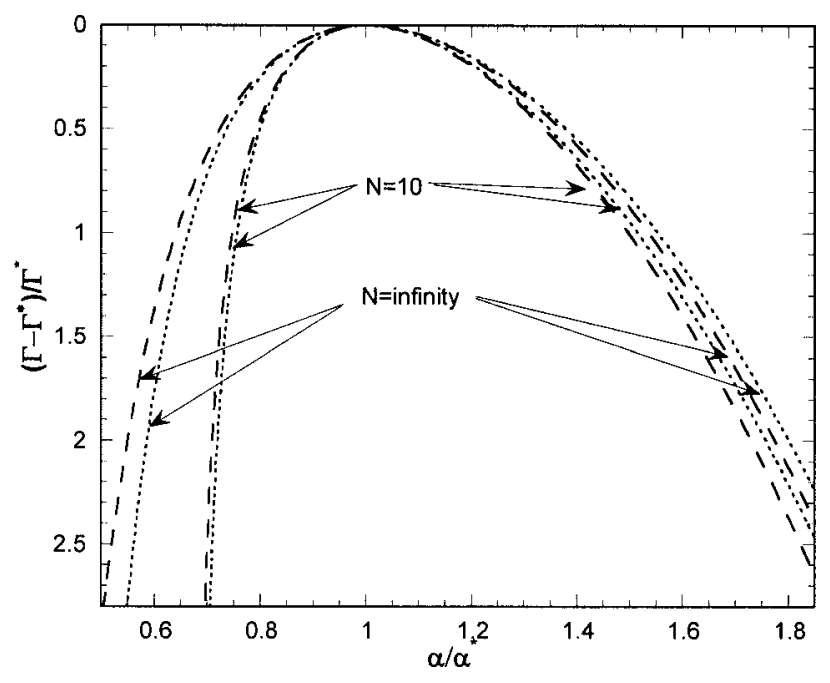

FIG. 8. Comparison of the phase boundary obtained from the equal free energy method, denoted by dotted lines, with that obtained from the Maxwell construction method, denoted by broken lines, for $N=10$ and $\infty$.

Hence, the envelope of the intramolecular phase segregation boundary calculated from the Maxwell construction method tends to shift towards smaller $\alpha$ compared to the intermolecular phase segregation boundary. Also, the magnitude of $\alpha$ may imply that for a given $\chi$, the intermolecular segregated globule state would have a more elongated structure than the spherical globule at a low monomer charge fraction (or $\Gamma$ ).

The phase boundaries obtained from the above two methods allow us to examine the dependence of chain length on conformational fluctuation. Figure 8 compares the phase boundary obtained from the equal free energy method, denoted by dotted lines, with that obtained from the Maxwell construction method, denoted by broken lines, for $N=10$ and $\infty$. Generally, the two methods show little difference at near their critical points. In the regime where $\alpha / \alpha^{*}>1$, the phase boundary computed from Maxwell construction shifts to smaller $\alpha$, and the magnitude of their shift is roughly the same for both chain lengths. For $\alpha / \alpha^{*}<1$, the boundary for the longer chain shifts more than that for the shorter chain. Moreover, the longer chain tends to have a wider phase boundary than the shorter chain. These findings indicate that a longer polyelectrolyte chain undergoes a greater size fluctuation, which may facilitate intramolecular phase segregation.

The effect of chain length on intramolecular and intermolecular phase segregations can be explored in a phase diagram. In the following, the calculation is proceeded for two different chain lengths at a fixed solvent quality, and the charge fractions to induce intramolecular and intermolecular phase segregations are determined. Figure 9 is the semilog plots of the charge fractions in terms of $\left(\Gamma=u f^{2} / 3\right)$ with swelling ratio $\alpha$ to identify the charge fraction calculated from the equal free energy method $(f)$, denoted by dotted broken lines, and from the Maxwell construction method $\left(f_{c}\right)$, denoted by solid lines, for $N=100$ and 1000 when $\chi$ $=0.7$; the phase boundaries of $N=1000$ obtained from the two methods are also plotted as references. The result shows that the phase boundary at small $\Gamma$ is insensitive to chain length. For the shorter chain $(N=100)$, the difference be- 


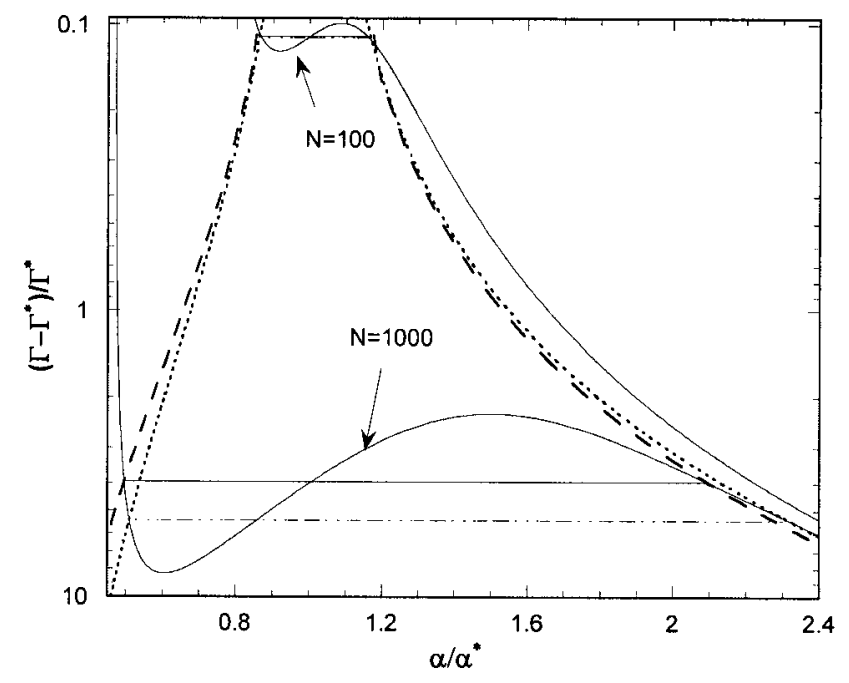

FIG. 9. Plot of the charge fractions (in terms of $\Gamma=u f^{2} / 3$ ) by using the equal free energy method $(f)$, denoted by dotted broken lines, and the Maxwell construction method $\left(f_{c}\right)$, denoted by solid lines for $N=100$ and 1000 when $\chi=0.7$. The phase boundaries of $N=1000$ obtained from the two methods are also plotted as reference; dotted line denotes the equal free energy method and broken line denotes the Maxwell construction method.

tween $f$ and $f_{c}$ is quite small, but for the longer chain $(N$ $=1000$ ), a significant difference between the two charge fractions is observed. Also, we notice that $f$ (or $\Gamma$ ) is greater than $f_{c}\left(\right.$ or $\Gamma_{c}$ ) for all cases, as the sample calculations shown in Fig. 9 [e.g., $\left(\Gamma-\Gamma^{*}\right) / \Gamma^{*}=5.42$ and $\left(\Gamma_{c}-\Gamma^{*}\right) / \Gamma^{*}=3.95$ for $N$ $=1000]$. These results may coincide with the previous studies in this area. First, the result of chain length dependent calculations qualitatively agrees with the recent simulation results in which the pearl necklace chain structures (due to intramolecular chain segregation) are usually more pronounced for longer chains. ${ }^{7,22}$ The explanation is given as follows. For shorter chains, intramolecular segregation occurs at the monomer charge fraction close to intermolecular segregation, and the conformations resulting from the two different phase segregation mechanisms may resemble to each other. For a longer chain, $f_{c}$ (or $\Gamma_{c}$ ) is significantly different from $f$ (or $\Gamma$ ), suggesting that the critical charge to induce intramolecular segregation is far away from that to induce intermolecular segregation. The intramolecular segregated conformational structures are expected to be very different from the intermolecular segregated conformational structures. Furthermore, the theory makes a prediction that $f>f_{c}$. This prediction, as shown in Figs. 7 and 9, is somehow consistent with the work by Iwaki and Yoshikawa. ${ }^{13}$ They concluded that the intramolecular segregation emerges when the monomer charge of a chain molecule is weak and the attractive interaction between monomers is present.

\section{CONCLUSIONS}

The diMarzio model has been extended to construct the phase diagram for the intermolecular and intramolecular conformational transitions of a single flexible polyelectrolyte chain in a dilute salt-free solution. Three variables are in the single chain free energy expression, including mean chain dimension, solvent quality, and monomer charge fraction, for a given chain length. At the long chain limit, the model re- sults in the same equation as that obtained from the more sophisticated chain model based on the Edwards Hamiltonian to deduce mean chain dimension. In the diMarzio theory, the phase boundary of intermolecular segregation is solved numerically by using the equal free energy method in which the two bistable (coexistent) conformations have the same free energy. Such a phase diagram exhibits the features of the first-order phase transition (like liquid-vapor phase diagram), consisting of both continuous and discontinuous transitions separated by a critical point. Under the discontinuous transition, the polyelectrolyte chain consists of coexistent (bistable) expanded and collapsed conformational states as seen in the experimental intermolecular phase segregation. Meanwhile, the theory falls into the universality class of mean field theory. Also, we find that the critical constants are sensitive to the chain length, given by $\left(\chi^{*}\right.$ $-1 / 2) \sim N^{-1 / 2}$ and $\Gamma^{*} \sim N^{-3 / 2}$. For the limiting long chain, the theory predicts that the mean chain size becomes a constant at the critical point and is roughly $90 \%$ of the mean size of an ideal chain. The result implies that partial contraction within a chain molecule is required to collapse a polyelectrolyte chain in the folding process. Besides, through the Maxwell equal-area construction method, the monomer charge fraction to induce intramolecular phase segregation (due to Rayleigh instability) is estimated. The findings show that for longer chains, the monomer charge fraction to induce intramolecular phase segregation is very different from that to induce intermolecular phase segregation, but for shorter chains, the difference becomes small. We speculate that the conformational structures arising from the intramolecular and intermolecular phase segregations differ significantly for longer chains, whereas the conformations involved in the two different phase segregation processes become similar for shorter chains. This result may account for the pronounced pearl necklace structures for long chain molecules observed in many simulation results. Furthermore, the theory predicts that the monomer charge fraction to induce intramolecular phase segregation is smaller than that to induce intermolecular phase segregation, consistent with the previous work in literature.

In addition to free energy and Maxwell construction, chemical potential is an alternative criterion in constructing phase boundary. The condition, at which intramolecular segregated conformations coexist with a globular or a coiled phase, may arise when these conformational states have the same chemical potential. Such a phase diagram will be investigated in our future work, along with the effect of explicit counterions and chain stiffness on the conformational behavior of a polyelectrolyte molecule.

\section{ACKNOWLEDGMENTS}

One of the authors (C.-Y.S.) received partial support for this work from the City University of New York PSC-CUNY Grant No. 67666-0036 and 68544-00-37, NSF Garcia MRSEC at SUNY Stony Brook, and NY START grant for the Center of Engineered Polymeric Materials at CSI and Institute of Macromolecular Assembly and also thanks Professor Arun Yethiraj for stimulating discussion and guidance. This 
study is partially supported by a Grant-in-Aid for Scientific Research for another author (K.Y.) from MEXT of Japan (8GS0421).

${ }^{1}$ P. J. Flory, Principle of Polymer Chemistry (Cornell University Press, Ithaca, NY, 1953).

${ }^{2}$ A. Y. Grosberg and A. R. Khohklov, Statistical Physics of Macromolecules (American Institute of Physics, New York, 1994).

${ }^{3}$ C. Williams, F. Brochard, and H. Firsch, Annu. Rev. Phys. Chem. 32, 433 (1981).

${ }^{4}$ P. G. Higgs and E. Raphael, J. Phys. I 1, 1 (1991).

${ }^{5}$ B.-Y. Ha and D. Thirumalai, Phys. Rev. A 46, R3012 (1992).

${ }^{6}$ C.-Y. Shew and A. Yethiraj, J. Chem. Phys. 110, 676 (1999).

${ }^{7}$ A. V. Lyulin, B. Dunweg, O. V. Bisov, and A. A. Darinskii, Macromolecules 32, 3264 (1999).

${ }^{8}$ K. Tsumoto, F. Luckel, and K. Yoshikawa, Biophys. Chem. 106, 23 (2003).

${ }^{9}$ A. Yamada, K. Kubo, T. Nakai, and K. Yoshikawa, Appl. Phys. Lett. 86, 223901 (2005).

${ }^{10}$ K. Yoshikawa, M. Takahashi, V. V. Vasilevskaya, and A. R. Khokhlov, Phys. Rev. Lett. 76, 3029 (1996)

${ }^{11}$ K. Yoshikawa, Adv. Drug Delivery Rev. 52, 235 (2001) and references therein.

${ }^{12}$ A. Kiriy, G. Gorodyska, S. Minko, W. Jaeger, P. Stepanaek, and M Stamm, J. Am. Chem. Soc. 124, 13454 (2002).

${ }^{13}$ T. Iwaki and K. Yoshikawa, Phys. Rev. E 68, 031902 (2003).

${ }^{14}$ T. Saito, T. Iwaki, and K. Yoshikawa, Europhys. Lett. 71, 304 (2005).

${ }^{15}$ S. Takagi, K. Tsumoto, and K. Yoshikawa, J. Chem. Phys. 114, 6942 (2001).

${ }^{16}$ M. Ueda and K. Yoshikawa, Phys. Rev. Lett. 77, 2133 (1996).
${ }^{17}$ K. Yoshikawa, Y. Yoshikawa, Y. Koyama, and T. Kanbe, J. Am. Chem. Soc. 119, 6473 (1997).

${ }^{18}$ Y. Yoshikawa, Y. Velichko, Y. Ichiba, and K. Yoshikawa, Eur. J. Biochem. 268, 2593 (2001).

${ }^{19}$ L. J. Kirwan, G. Papastavrou, M. Borkovec, and S. H. Behrens, Nano Lett. 4, 149 (2004).

${ }^{20}$ D. Baigl, M. Sferrazza, and C. E. Williams, Europhys. Lett. 62, 110 (2003).

${ }^{21}$ D. Qu, D. Baigl, C. E. Williams, H. Mohwald, and A. Fery, Macromolecules 36, 6878 (2003).

${ }^{22}$ A. V. Dobrynin, M. Rubinstein, and S. P. Obukhov, Macromolecules 29, 2974 (1996).

${ }^{23}$ F. J. Solis and M. O. de la Cruz, J. Chem. Phys. 31, 5502 (1998).

${ }^{24}$ H. J. Limbach, C. Holm, and K. Kremer, Europhys. Lett. 60, 566 (2002).

${ }^{25}$ H. J. Limbach and C. Holm, J. Phys. Chem. B 107, 8041 (2003).

${ }^{26}$ Y. Kantor and M. Kardar, Phys. Rev. E 51, 1299 (1995).

${ }^{27}$ S. F. Edwards, Proc. Phys. Soc. London 85, 613 (1965).

${ }^{28}$ T. P. Lodge and M. Muthukumar, J. Phys. Chem. 100, 13275 (1996).

${ }^{29}$ Y. Chen and A. L. Kholodenko, J. Chem. Phys. 86, 1540 (1987).

${ }^{30}$ M. Muthukumar, J. Chem. Phys. 86, 7230 (1987).

${ }^{31}$ A. Dua and T. A. Vilgis, Europhys. Lett. 71, 49 (2005).

${ }^{32}$ T. A. Vilgis, A. Johner, and J. F. Joanny, Eur. Phys. J. E 2, 289 (2000).

${ }^{33}$ E. A. diMarzio, Macromolecules 17, 969 (1984).

${ }^{34}$ E. A. diMarzio and C. M. Guttman, J. Phys. Chem. 93, 7004 (1989).

${ }^{35}$ P.-G. deGennes, P. Pincus, R. M. Velasco, and F. Brochard, J. Phys. (France) 37, 1461 (1976).

${ }^{36}$ Y. Chen and C.-Y. Shew, J. Chem. Phys. 115, 9084 (2002).

${ }^{37}$ J. M. Yeomans, Statistical Mechanics of Phase Transitions (Oxford Science, Oxford, 1992).

${ }^{38}$ T. Sakaue and K. Yoshikawa, J. Chem. Phys. 117, 6323 (2002).

${ }^{39}$ N.-K. Lee and S. Obukhov, Europhys. Lett. 66, 350 (2004). 